\title{
Varja \\ Susciter le développement professionnel en étayant la réflexivité en formation continue : une étude de cas à l'école maternelle
}

\author{
Stéphane Talérien, Université de La Réunion, Sébastien Chaliès, Université de Toulouse 2 et \\ Stefano Bertone, INSPÉ-Université de La Réunion
}

Cette étude de cas propose, à partir d'un programme de recherche anthropoculturel, un dispositif de formation adossé à la recherche qui crée les conditions formelles propices à la transmission efficace des règles d'une pratique pédagogique ordinaire entre deux enseignantes expérimentées exerçant au sein de la même école maternelle. Afin de produire leur développement professionnel, les deux enseignantes (l'une tenant le rôle de "pair formateur " et l'autre celui de "pair formé") ont vécu trois épisodes de formation: (i) l'accès préalable du pair formateur à une vision plus claire des règles régissant sa pratique devant être transmise (ii) l'enseignement ostensif de ces règles par le pair formateur en direction du pair formé et (iii) deux mises en æuvre par le pair formé suivies à chaque fois d'une activité d'accompagnement sous forme de contrôle du pair formateur. Les résultats obtenus montrent (a) l'apprentissage d'une nouvelle règle par le pair formé lui permettant de résoudre une difficulté professionnelle ainsi que (b) le développement professionnel du pair formateur suite à son accompagnement de la pratique du pair formé.

\section{Introduction}

En France, la loi pour une École de la confiance de juillet 2019 a abaissé l'instruction obligatoire à l'âge de trois ans. Cette décision a eu pour effet d'affirmer l'importance de l'école maternelle dans la réussite de la scolarité des élèves (Suchaut, 2008). Mais si l'enseignement pré-élémentaire tient une place de choix dans le système éducatif français, il est possible de s'interroger sur la qualité de la formation des professeur.e.s des écoles exerçant à l'école maternelle.

En effet, d'un côté la formation initiale des enseignant.e.s du premier degré accorde peu de place aux spécificités de l'école maternelle (Inspection générale de l'éducation nationale [IGEN], 2017) ou traite ses problématiques propres de façon éclatée et ramenée aux autres disciplines d'enseignement de l'élémentaire (Bouysse, 2018) ; et de l'autre, les possibilités de développement professionnel par (a) la formation continue et par (b) la réflexivité (Schön, 1994) individuelle et/ou collaborative se heurtent à plusieurs écueils.

(a) La formation continue des enseignant.e.s en France se caractérise surtout par la participation à des cours, des séminaires ou à des conférences (OCDE, 2019). Or, ces formats " traditionnels » de formation (Stein et al., 1999) placent les enseignant.e.s dans une posture de consommation de connaissances « exogènes » (Little, 1993) produites à l'extérieur de l'établissement scolaire. La formation continue est alors trop fragmentée, éloignée de la pratique réelle des enseignant.e.s en classe et non alignée avec les théories actuelles de l'apprentissage (Borko et al., 2011). Au final, ce que les enseignant.e.s apprennent dans ces formats traditionnels de formation n'est pas suffisamment transféré dans la pratique quotidienne (Fraser, 2010 ; Poulson et Avramidis, 2003).

(b) La réflexivité individuelle des enseignant.e.s sur leurs pratiques est malaisée. Elle ne consiste souvent qu'en une reviviscence superficielle de certaines situations du travail ou en la mise en œuvre de stratégies de résolution des problèmes professionnels pouvant consister à les minorer, voire à les ignorer (Consuegra et al., 2014). Lorsqu'il relève de la seule réflexivité individuelle, l'apprentissage au travail peut par ailleurs conduire à l'appropriation de "mauvaises leçons" et/ou habitudes (Bell et Dale, 1999). Par ailleurs, bien que certains modèles tels que "les communautés d'apprentissage professionnel » ou "l'étude collective de leçon " mettent en avant les intérêts de la réflexivité collaborative pour soutenir le développement professionnel (Stoll et al., 2006 ; Vescio et al., 2008), certains travaux sont plus nuancés. L'effet de ce type de réflexivité est alors qualifié de "sommaire, faible, mais significatif " (Lomos et al., 2011) ou de "subtil et peu marqué » (Ermeling et Yarbo, 2016).

Afin d'optimiser la formation continue et de soutenir la réflexivité des enseignant.e.s au sein des établissements scolaires, des études récentes (Ermeling et Yarbo, 2016 ; Postholm, 2018) mettent avant le caractère fécond de partenariats établis entre la communauté de professionnel.le.s et des intervenant.e.s extérieur.e.s tels que les chercheur.se.s universitaires. En France par exemple, en s'appuyant sur l'organisation spécifique du secondaire, 
les laboratoires pour le changement (Ria et Moussay, 2014) permettent d'impulser le développement de nouvelles manières d'agir et d'accompagner les enseignant.e.s dans le renouvellement de leurs pratiques. Au sein de ce type de partenariat, le rôle des chercheur-se.s consiste donc à provoquer et soutenir un processus de développement professionnel mené par et pour les praticien·ne.s (Postholm, 2018). Les études s’inscrivant dans un tel partenariat ont en commun de susciter la réflexivité individuelle et collaborative des enseignant.e.s à partir de traces réelles d'activité (Félix, 2014 ; Moussay et Blanjoie, 2016 ; Ria et Lussi Borer, 2015). Dans cette perspective, les chercheur-se.s favorisent ce retour réflexif par l'utilisation de différents " outils " tels que l'observation directe (Ermeling et Yarbo, 2016) ou audiovisuelle (González et al., 2016) des pratiques d'enseignement, par l'analyse des résultats d'élèves (Wood et al., 2017) ainsi que par le dialogue entre enseignant.e.s (Grau et al., 2017) ou entre les chercheur.se.s et les enseignant.e.s (Wood et al., 2017). Alors que toutes ces études considèrent que le seul engagement de la réflexivité " outillée " peut conduire au développement professionnel, une zone d'ombre subsiste toutefois au sein de cette littérature scientifique. Rares sont effectivement encore à ce jour les travaux qui documentent des changements effectifs dans la pratique d'enseignement imputables à des analyses réflexives collaboratives menées dans le cadre d'un partenariat entre une communauté d'enseignant.e.s et un·e ou plusieurs chercheur.se.s universitaires.

L'objet de cet article porte précisément sur les effets en matière de développement professionnel d'un dispositif de formation qui s'inscrit dans tel partenariat. Les enseignant.e-s d'une école maternelle nous ont exprimé leur difficulté à se transmettre des pratiques professionnelles ordinaires jugées par eux ou elles comme efficaces et nous avons exprimé aux enseignant.e.s notre souhait de produire des connaissances scientifiques sur leur activité professionnelle au moyen d'un dispositif « utile " pour résoudre leur problème professionnel. Sera ainsi étudié dans le détail le cas de la transmission explicite et de la transformation d'une pratique ordinaire d'enseignement entre deux enseignantes expérimentées exerçant au sein de la même école maternelle par le truchement d'un dispositif de formation continue adossé à la recherche.

\section{Choix théoriques et implications technologiques}

Cette étude de cas s'inscrit dans un Programme de Recherche Technologique (PRT) anthropoculturel (Bertone et Chaliès, 2015) principalement inspiré par la philosophie du langage ordinaire de Wittgenstein (2004). Mobilisant une épistémologie lakatossienne (Lakatos, 1994), ce PRT propose une conceptualisation de la formation professionnelle à partir d'un noyau dur théorique et d'une série d'hypothèses auxiliaires. À l'interface de la formation et de la recherche, ces hypothèses permettent de réaliser des observations scientifiques et consubstantiellement de tester la fécondité du dispositif de formation mis en œuvre.

Dans le cadre de la présente étude, l'hypothèse auxiliaire incarnée au sein du dispositif était la suivante : "La transmission explicite entre enseignant.e.s expérimenté·ess des règles régissant leurs pratiques professionnelles ordinaires est susceptible de favoriser leur apprentissage de ces règles ainsi que leur développement professionnel à partir du suivi interprété de celles-ci ». Sur la base de cette hypothèse, un dispositif de formation continue a été conçu de telle sorte qu'un.e enseignant.e expérimenté.e maîtrisant une pratique spécifique (correspondant à un·e pair·e formateur-trice - PFR) puisse enseigner explicitement les règles régissant cette pratique à un·e autre enseignant·e expérimenté·e ne les maîtrisant pas encore (correspondant à un·e pair·e formé·e - PFÉ).

La présentation théorique qui suit décrit les principaux postulats retenus permettant d'expliciter l'hypothèse préalablement délimitée et détaille le contenu du dispositif de formation mis en œuvre notamment en matière d'activités déployées par les acteur·rice.s.

\section{Étape 1 : Permettre au PFR d'accéder à La vision synoptique de sa pratique spéc if ique}

Selon Wittgenstein, «suivre une règle est une pratique » $(2004, \$ 202)$. Autrement dit, la pratique professionnelle spécifique du PFR peut être assimilée à la réalisation d'actions conformes à des suivis de règles spécifiques. L'enseignement de ces règles par le PFR en direction du PFÉ suppose son propre accès à ces règles. Or, cet accès de l'acteur-rice aux règles régissant sa propre pratique est rendu malaisé par l'existence d'un double régime de réflexivité (Ogien, 2007) : une réflexivité de l'ordre de « l'inhérence » et une réflexivité de l'ordre de «l'appréhension de l'inhérence ". Dans le cas du régime de réflexivité de l'ordre de l'inhérence, "la réalisation d'un acte est son intention" (Ogien, 2007, p. 51). Dans ce cas de figure, l'action est gouvernée par des règles et l'acteur·rice agit selon ces règles. Ces dernières sont alors soit préconscientes, mais potentiellement dicibles, car apprises lors d'une formation réflexive, soit non conscientes, car apprises de façon implicite par participation à des pratiques 
partagées au sein de la communauté (Lave et Wenger, 1991). Dans ce dernier cas, elles ne sont donc dicibles que moyennant une prise de conscience ultérieure lors d'une situation dialogique à propos des actions réalisées. La réflexivité de l'ordre de l'appréhension de l'inhérence s'enracine dans ces dialogues qui rendent possible l'appréhension du lien logique entre l'intention et l'action. Au cours de ces dialogues, l'acteur.rice apprend finalement à énoncer une règle et/ou à l'invoquer pour justifier le sens de son action passée ou à venir.

Cette première proposition théorique s'est traduite par des décisions technologiques visant à étayer la réflexivité des enseignant.e.s. En effet, dans la perspective de l'enseignement des règles constitutives de sa pratique de classe au PFÉ, le PFR l'a d'abord filmée puis a vécu un dialogue avec le chercheur sous la forme d'un entretien d'autoconfrontation (EAC) à propos des actions suivies. Le PFR a pu ainsi expliciter ces règles qui régissaient sa pratique en instruisant le chercheur sur la signification des actions observées. Lors de cette instruction, le PFR a donc pu énoncer les règles ordinaires qu'il avait suivies dans sa pratique de classe. Cet aménagement technologique a ainsi permis au PFR d'accéder à une vision plus claire, synoptique, des règles sous-jacentes à sa pratique de classe et de s'engager ultérieurement dans leur enseignement ostensif au PFÉ.

Étape 2 : Enseigner ostensivement la pratique spécifique au PFÉ

Une autre décision technologique a été de demander au PFR de se livrer ensuite à une activité d'enseignement ostensif (Wittgenstein, 2004) des règles régissant sa pratique. Pour ce faire, le PFR a fondé pour chacune des règles objet d'enseignement un lien de signification entre (a) l'expérience langagière d'énonciation de la règle, (b) les circonstances expérientielles identifiées et montrées ostensivement y étant associées et (c) les résultats pouvant être obtenus dans le cas d'un suivi adéquat de la règle préalablement enseignée. Par exemple, le PFR a enseigné au PFÉ la règle (a) étiquetée "Utiliser une voix spécifique pour la marionnette ", (b) en lui montrant les circonstances expérientielles associées à cette règle " il y a des moments où c'est Bernie qui parle, et il y a des moments où c'est moi qui parle ", et (c) en lui spécifiant ce que le suivi correct de cette règle devrait obtenir comme résultat "permettre aux élèves de faire la part des choses entre le moment où c'est la marionnette qui parle et où c'est toi qui parles".

Pour mener à bien cet enseignement, le PFR s'est notamment appuyé sur l'enregistrement vidéo de sa pratique de classe (Talérien et Bertone, 2018). Concrètement, pour chacune des actions visionnées et significatives pour le PFR ou le PFÉ, la vidéo a été arrêtée, une signification lui a été attribuée et l'attente de résultats associés à cette action a été verbalisée.

\section{Étape 3 : Mettre en œuvre une première fois et contrôler-accompagner ce premier usage}

Lors de cette troisième étape, le PFÉ s'est engagé au sein de sa classe dans les premiers suivis des règles préalablement enseignées par le PFR. Ce dernier a accompagné le PFÉ dans ce premier usage. Cet accompagnement a consisté à " contrôler " (Nelson, 2008) le caractère adéquat du suivi des règles. Lorsque cela s’est révélé nécessaire, c'est-à-dire lorsque la pratique observée était considérée comme inadéquate ou porteuse de malentendus, l'accompagnement a alors consisté pour le PFR à procéder à des « explications ostensives » (Wittgenstein, 2004) en reprenant son enseignement et en multipliant les exemples pouvant être associés à chacune des règles.

L'activité de contrôle-accompagnement du PFR s'est déroulée lors d'un entretien post-leçon à partir de l'enregistrement audio-vidéo de la pratique de classe du PFÉ. Les premiers usages en classe et l'entretien post-leçon qui a suivi, ont ensuite permis au PFÉ de s'engager dans une seconde mise en œuvre.

\section{Étape 4 : Mettre en œuvre une seconde fois et contrôler-accompagner ce second usage}

La seconde mise en œuvre était pour le PFÉ le moment de faire sienne la pratique spécifique du PFR en procédant notamment aux modifications qui lui semblaient pertinentes.

Le second usage était donc l'occasion d'une interprétation des règles par le PFÉ. À l'origine de ce développement de l'activité du PFÉ, a donc été provoquée une interprétation des règles en tant que «substitution d'une expression de la règle à une autre" (Wittgenstein, 2004, \$201). Cette substitution correspondait à un usage " extensif " des règles enseignées (Chaliès et Bertone, 2008).

Au niveau du dispositif de formation, suite aux premières explications livrées par le PFR lors de l'entretien post-leçon consécutif à la première mise en ouvre, le PFÉ a pu effectuer un nouvel usage de la pratique spécifique auprès de ses élèves. Il avait en outre pour consigne d'essayer de "s'éloigner ", s'il le souhaitait, de la pratique spécifique enseignée par le PFR en mettant cette pratique "à sa main ». Cette seconde réalisation était également filmée. Suite à celle-ci, le PFR s'est à nouveau livré à un entretien post-leçon avec le PFÉ. Il a une

2021 RSSE 42 (3), DOI $10.24452 / \mathrm{sjer.43.1.12}$ 
nouvelle fois essayé de l'accompagner, cette fois-ci dans son développement, en s'appuyant sur l'enregistrement audio-vidéo.

\section{Méthode}

\subsection{Le dispositif de formation support à l'étude et ses participants}

Le dispositif de formation correspond à la transformation (Bertone et Chaliès, 2015) des activités d'apprentissage informel réalisées par deux enseignantes lors de l'année scolaire 2015-2016. L'hypothèse auxiliaire incarnée par le dispositif est indexée au programme de recherche anthropoculturel. À ce titre, le dispositif s'est appuyé sur une démarche de recherche dite "transformative " (Clot, 2008 ; Theureau, 2006) qui a consisté à modifier le contexte de travail des acteur-rice-s ainsi que leurs activités afin de mieux les étudier et susciter des transformations fécondes. Par rapport à des activités d'apprentissage informel de type collaboratif avec récit des expériences de classe (Little, 2007), et sur la base des postulats théoriques énoncés antérieurement, le dispositif de formation était structuré autour de quatre changements correspondants aux quatre activités singulières de formation présentées précédemment.

Les deux participantes étaient des enseignantes expérimentées exerçant au sein de la même école maternelle à l'Académie de La Réunion. Le PFR était une enseignante expérimentée de 48 ans et avait 20 ans d'expérience professionnelle. Elle ${ }^{1}$ était titulaire du CAFIPEMF (Certificat d'aptitude aux fonctions de professeur des écoles maître formateur), était rompue à l'exercice de la formation d'enseignant.e.s et/ou d'enseignant.e.s stagiaires et avait suivi elle-même une formation sur le conseil en formation de terrain. Le PFÉ pour sa part était une enseignante expérimentée âgée de 37 ans et avait 10 ans d'expérience professionnelle. Elle n'avait pas à proprement parler d'expérience de formation.

Les enseignantes avaient chacune une classe de cycle, les enfants de leurs classes avaient entre trois et cinq ans et étaient en petite, moyenne et grande sections. Les deux salles de classe étaient mitoyennes. Leurs préoccupations portaient sur la présentation des ateliers d'apprentissage aux élèves. Le PFR pratiquait depuis de nombreuses années une activité consistant à induire les activités d'apprentissage : le matin après une séance d'activité physique, le PFR utilisait une marionnette (Bernie) afin de présenter les ateliers de la matinée. Elle avait décidé d'appeler ce moment "Bernie passeur de savoirs ". Lors de cette activité, le PFR insistait auprès de ses élèves sur ce qu'ils et elles allaient apprendre et sur les lieux au sein de la classe où allaient se dérouler les ateliers. À la fin de ce moment, les élèves se dirigeaient de façon volontaire et autonome vers les activités qu'ils et elles souhaitaient effectuer. Par le passé, le PFÉ s'était déjà montrée intéressée par cette pratique du PFR. D'ailleurs, elle avait essayé de la mettre en place dans sa classe en utilisant elle aussi une marionnette qu'elle avait appelée "Épic ». Mais n’ayant pas obtenu de résultats satisfaisants, elle avait dû renoncer. C'est donc dans la perspective de mieux transmettre cette pratique pour l'une et de mieux se l'approprier pour l'autre, que le PFR et le PFÉ se sont engagées dans le présent dispositif.

\subsection{Le recueil et L'analyse des données}

Deux catégories de données ont été recueillies : (a) les données d'enregistrement du travail : afin de documenter au mieux les pratiques, les enseignantes avaient capturé leurs actions au moyen de deux petites caméras d'action, et (b) les données d'entretien d'autoconfrontation (EAC). Afin de recueillir les significations attribuées par les actrices à leurs différentes actions lors de chaque étape du dispositif, chacune des étapes avait fait l'objet d'EAC d'une durée approximative de une à deux heures après la leçon et sur le temps de classe (9 EAC ont ainsi été réalisés). Par ailleurs, le questionnement semi-structuré du chercheur devait permettre aux enseignantes d'énoncer les règles identifiées et/ou de les aider à formuler ces règles (pour chacune des actions, les questions principales étaient " que fais-tu ?", " qu'est-ce que tu en penses ? " et "pour quelle(s) raison(s) fais-tu cela ? "). Le chercheur incitait également les actrices à opérer des reformulations lorsque cela était nécessaire et engageait parfois une controverse afin de lever les éventuelles incompréhensions de sa part et accéder à un maximum d'éléments d'étayage de l'exemple visionné.

Les données de l'enregistrement audio-vidéo des EAC ont été retranscrites verbatim afin de pouvoir identifier les règles suivies par les enseignantes. Dans le but de formaliser ces règles, l'ensemble de la retranscription verbatim a été traitée en trois étapes successives (Bertone, 2016).

$\overline{1}$ Le PFR et le PFÉ étant des enseignantes, dans la suite du texte le choix d'utiliser une chaîne anaphorique au féminin a été fait. 
(i) Le corpus a été découpé en unités d'interaction. Ces unités correspondaient aux objets de signification attribuée par l'actrice (ces objets de signification étaient par exemple : "chanter la chanson de Bernie ", "capter l'attention des élèves", "changer de voix ").

(ii) Pour chaque objet de signification, l'étayage apporté par l'actrice a été identifié et associé à l'ensemble des circonstances qu'elle avait énoncées pour expliquer au chercheur comment signifier comme elle les actions visionnées dans ces circonstances. Par exemple, pour tel objet de signification, l'actrice a pu expliquer au chercheur " ce que je vois là s'intitule ainsi, car j’y vois ces aspects-là qui relèvent de ce à quoi correspond ce que je viens de t'intituler ou encore des résultats que je peux constater ou pas dans ce qui est fait ".

(iii) Pour chaque unité d'interaction, une expression de la règle mobilisée a été formalisée. Par convention, chaque règle a été étiquetée à partir (a) de l'objet de la signification attribuée par l'actrice, (b) de l'ensemble des éléments évoqués par l'actrice pour étayer cette signification et (c) des résultats constatés et/ou attendus. Chaque règle a été formalisée avec la syntaxe suivante ["Objet de la signification » vaut pour les éléments où "ensemble des éléments évoqués pour étayer la signification " ce qui obtient comme résultat "ensemble des résultats constatés etlou attendus»]. À titre d'exemple [(chanter) "la chanson » vaut pour "introduire l'arrivée de Bernie (la marionnette) de façon ritualisée " ce qui obtient pour résultats "de marquer ce moment-là de la journée ", "d'attirer l'attention de chacun»].

Des enquêtes grammaticales diachronique et synchronique ont enfin été menées. L'enquête grammaticale diachronique a été réalisée pour retracer l'historicité des règles apprises et/ou suivies par les actrices sur l'ensemble des étapes du dispositif de formation. Une enquête grammaticale synchronique a ensuite été réalisée pour comparer les règles suivies par chacune des actrices lors d'une même étape du dispositif de formation. Par convention, nous avons considéré que les actrices suivaient la même règle si l'objet de signification, certains éléments d'étayage et les résultats y étant associés étaient identiques.

\section{Résultats}

Les deux résultats détaillés ci-après ont été obtenus dans le cadre de cette étude de cas. Ils nécessitent d'être corroborés et consolidés par une casuistique plus importante. Néanmoins, ils permettent dans le cadre de cet article de mettre en exergue le caractère heuristique de l'hypothèse auxiliaire qui était délimitée comme suit : " La transmission explicite entre enseignant.e-s expérimenté·e·s des règles régissant leurs pratiques professionnelles ordinaires est susceptible de favoriser leur apprentissage de ces règles ainsi que leur développement professionnel à partir du suivi interprété de celles-ci ".

Les deux résultats détaillés concernent (i) l'apprentissage d'une nouvelle règle par le PFÉ relative à l'utilisation de la voix, et (ii) le développement professionnel de l'activité dialogique du PFR suite au dialogue sur la pratique du PFÉ.

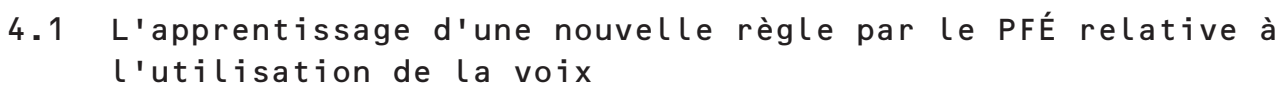

La pratique spécifique du PFR "Bernie passeur de savoirs " mobilisait une marionnette nommée "Bernie ", mais également une voix spécifique pour ce personnage.

Lors de l'EAC sur sa pratique, le PFR avait expliqué au chercheur que jouer une voix spécifique pour la marionnette permettait aux élèves de distinguer Bernie de la maîtresse et d'être attiré.e.s par ce personnage qui a un rôle différent de celui de l'enseignante (Extrait 1).

Extrait 1 PFR - EAC - Pratique spécifique "Bernie passeur de savoirs »

\section{Notes d'observation du chercheur sur la pratique spécifique du PFR à partir de l'enregistrement vidéo}

La marionnette Bernie s’adresse aux élèves. La maittresse joue une voix différente.

\section{Extrait de l'EAC}

Chercheur (32'34") : donc là tu changes de voix, tu gardes ta voix, tu...

PFR : je change de voix. Il y a Bernie et il y a moi. Ça aussi c'est important. Des fois je m’emmêle un peu les pinceaux. On le voit plus tard... (rire)... mais hop, je ramène. Mais ils savent. Ils savent que c'est moi. Ils savent que c'est ma main qui est dans la marionnette, mais... Mais il est beaucoup plus attractif que moi. Il a sa propre voix, et il a un rôle aussi différent du mien. Ce n’est pas la maîtresse. 
Au cours de cet extrait, le PFR a rendu compte de son activité en énonçant la règle ["je change de voix » vaut pour " (jouer une voix différente pour Bernie) " ce qui obtient pour résultats "Il y a Bernie et il y a moi", "Il (Bernie) a sa propre voix ", "il est beaucoup plus attractif que moi ", "il a un rôle aussi différent du mien "]. Lors du temps de formation du PFÉ, le PFR a enseigné cette règle au PFÉ (Extrait 2).

\section{Extrait 2 Enseignement ostensif PFR - PFÉ}

PFR (3'22") : donc là aussi, Bernie sort de sa maison, ils disent bonjour à Bernie. Pareil, il est là. On capte l'attention sur Bernie, en lui disant bonjour, lui dit bonjour et on lui répond.

PFÉ : mais tu ne changes pas ta voix ?PFR : oui, un peu. Si, si.

PFÉ : d'accord.

PFR : oui il y a des moments où c'est Bernie qui parle, et il y a des moments où c'est moi qui parle. On est en fait associé lui et moi.

PFÉ : d'accord, donc tu prends une voix pour Bernie.

PFR : oui. [...]

PFÉ : non, mais c'est juste pour savoir si j'ai besoin de changer l'intonation. Puisque moi avec Épic j’ai la même chose. J'ai Épic, pareil, mais c'est ma voix finalement. Et les enfants me disent : "maîtresse, pourquoi c'est pas Épic qui parle et c'est toi qui parles? " Parce qu'Épic n'a pas de voix, je suis obligée de parler pour lui. PFR : du coup, ils ne font pas la part des choses entre le moment où c'est Épic qui parle et où c'est toi qui parles.

Lors de cet échange, le PFR a enseigné au PFÉ la règle [" (utiliser une voix spécifique pour Bernie) » vaut pour "il $y$ a des moments où c'est Bernie qui parle, et il y a des moments où c'est moi qui parle " ce qui obtient pour résultat "(de permettre aux élèves de faire) la part des choses entre le moment où c'est Épic qui parle et où c'est toi qui parles"].

Alors que le PFR a arrêté la vidéo afin d'expliquer la règle relative au réveil de Bernie " donc là aussi, Bernie sort de sa maison, ils disent bonjour à Bernie », le PFÉ l'a interrogée sur la voix de la marionnette. À cette occasion, le PFR a enseigné d'abord la règle se rapportant à l'utilisation d'une voix spécifique pour la marionnette et a expliqué l'importance de distinguer les moments où la marionnette parle pour que les élèves ne confondent pas les voix ("ils ne font pas la part des choses entre le moment où c'est Épic qui parle et où c'est toi qui parles").

Cet extrait montre également la préoccupation du PFÉ quant à l'utilisation d'une voix spécifique pour la marionnette (" c'est juste pour savoir si j’ai besoin de changer l'intonation. Puisque moi avec Épic (...) c'est ma voix finalement. Et les enfants me disent : "maîtresse, pourquoi c'est pas Épic qui parle et c'est toi qui parles?"). Cette préoccupation provient d'une tentative infructueuse du PFÉ, antérieure à la présente situation de formation, visant à utiliser la marionnette sur la base d'un apprentissage informel. C'est d'ailleurs pour mieux mettre en œuvre cette pratique au sein de sa classe que le PFÉ s'est engagée dans ce dispositif de formation.

Lors du premier usage, le PFÉ a utilisé une voix spécifique pour sa marionnette (Épic) et a constaté notamment un dialogue entre Épic et ses élèves (Extrait 3).

Extrait $3 P F E-E A C-$ Premier usage

\section{Notes d'observation du chercheur sur la pratique du PFÉ à partir de l'enregistrement vidéo}

La classe a fini de chanter la chanson d'Épic, l'enseignante prend en main la marionnette. Épic fait mine de se réveiller puis s'adresse aux élèves «Bonjour les enfants ! " Un élève dit "Ça fait longtemps qu'on ne t'avait pas vu Épic! » La marionnette répond « Oui dis-donc, ça fait un week-end. » Un autre élève dit alors "Pourquoi tu parles comme ça Épic?"

\section{Extrait de l'EAC}

PFÉ (4'52") : question d'Odile "pourquoi je parle comme ça ? " Effectivement à un certain moment, je ne sortais plus beaucoup Épic, donc c'est vrai que... il n'avait pas vraiment de voix. Et puis je mélangeais vachement. Là j’ai pris vraiment " la voix d'Épic ». On va essayer de la garder cette voix... plus grave, un peu plus... rauque. Voilà, avant je mélangeais de trop. C'est vrai qu'ils se perdaient aussi peut-être dedans les enfants.

Chercheur : d'accord, donc là tu dis que le fait de prendre cette voix-là, ça apporte quoi ? Ça fait que...

PFÉ : ben là, ils s'adressent vraiment à Épic. Ils ne me voient plus moi. Ils s'adressent vraiment... enfin dans la suite de la vidéo... ils se sont vraiment adressés à Épic. Ils n’ont pas demandé maîtresse. Vraiment à Épic. 
Chercheur : donc tu dis que le fait de changer de voix fait qu'ils peuvent s'adresser à Épic.

PFÉ : ils s'adressent à Épic. Ils connaissent sa... voilà, par rapport à la voix, c'est Épic qui parle. Si c'est une autre voix ce sera maîtresse qui parlera. Avant c'était un peu plus mélangé tu vois. C'était vraiment... ben comme ils n'arrêtaient pas de le toucher à chaque fois. Donc du coup... ben c'est toi, c'est moi, c'est toi, c'est moi... enfin bref. Là, tu vois, maintenant le fait de poser les choses, ils sont beaucoup plus calmes, ils sont beaucoup plus attentifs et du coup ça me permet d'être beaucoup plus attentive dans ce que je fais finalement. Et là, Leïla me demande " maîtresse tu as changé de voix ? " Ben oui... bon après, j'étais grippée aussi... donc la voix n'était pas forcément... voilà. Mais je vais garder une voix rauque. Avant c'était une voix mi- rauque, mi- moi ! [...]

Chercheur : tu es vraiment satisfaite du fait qu'ils s'adressent vraiment à Épic ?

PFÉ : ah oui ! Ah ben oui ! Au moins il n'y a plus ce moment où tout est mélangé. C'est vraiment ce moment... le moment Épic est là. Et là, oui moi, ça m’a changé.

Chercheur : ah oui, carrément.

PFÉ : ah ça m’a changé. Avant c'était «Épic je te fais des bisous... » il n’avait même pas le temps de parler le pauvre Épic. Il était complètement débordé par des tonnes de câlins. Là, ils sont calmes, après on avait réexpliqué les choses vendredi déjà... " il va avoir une maison, on va chanter, on va attendre il faudra être patient, et tout ça..." là ils le sont.

Chercheur : ils sont patients, et puis ils s'adressent à Épic.

PFÉ : la voix, ça y est, ça résonne. La voix, c'est une autre voix que celle de maîtresse.

Au cours de cet extrait, le PFÉ a rendu compte de son activité en énonçant la règle ["j’ai pris vraiment la voix d'Épic " vaut pour " (utiliser) cette voix... plus grave, un peu plus... raqque" ce qui obtient pour résultats "ils s'adressent vraiment à Épic ", "Ils ne me voient plus moi ", "ils sont beaucoup plus calmes, ils sont beaucoup plus attentifs et du coup ça me permet d'être beaucoup plus attentive dans ce que je fais finalement "].

Le PFÉ a rendu compte au chercheur de son propre usage de la voix d'Épic (" on va essayer de la garder cette voix... plus grave, un peu plus... rauque ») et du fait que l'utilisation de cette voix permettait d'obtenir des résultats différents. Les élèves (a) en oubliaient la présence de la maîtresse ("ils ne me voient plus moi ») au point de s'adresser à la marionnette ("ils se sont vraiment adressés à Épic »), (b) étaient calmes et attentifs ("ils sont beaucoup plus calmes, ils sont beaucoup plus attentifs»), (c) ce qui permettait corollairement à l'enseignante d'être à son tour plus attentive à sa propre pratique (" ça me permet d'être beaucoup plus attentive dans ce que je fais finalement").

En se conformant à l'activité du PFR, l'enseignante a réussi à dissocier ses prises de parole (avec sa propre voix) de celles de la marionnette (avec la voix d'Épic) et ainsi permis aux élèves de faire la part des choses entre le rôle de la maitresse et celui de la marionnette. Les résultats montrent un apprentissage effectif de cette règle par le PFÉ, qui a réussi non seulement à agir conformément aux explications ostensives de PFR, mais a considéré également avoir réglé un problème antérieur de cette pratique. En effet, l'enseignante a précisé qu’à la suite d'un apprentissage informel, elle n'avait pas vraiment octroyé une voix spécifique à la marionnette ("il n'avait pas vraiment de voix. Et puis je mélangeais vachement") ce qui avait pour effet néfaste que les élèves "se perdaient " et "n'arrêtaient pas de le toucher (Épic) à chaque fois ". Les élèves étaient alors davantage ancré.e.s sur la peluche que sur le personnage vecteur d'apprentissage ce qui avait pour effet que "le pauvre Épic (...) était complètement débordé par des tonnes de câlins". L'utilisation de la marionnette était donc devenue, dans la pratique antérieure du PFÉ, plus un objet de distraction que de concentration sur la présentation des ateliers de travail. L'apprentissage de cette règle par le PFÉ, suite à l'enseignement ostensif réalisé par le PFR, a engendré un développement satisfaisant d'une pratique antérieure et a contribué à résoudre un problème professionnel.

\subsection{Le développement professionnel de L'activité dialogique du PFR suite} au dialogue sur la pratique du PFÉ

La pratique spécifique "Bernie passeur de savoirs" s'appuyait également sur l'utilisation d'un "panneau des apprentissages ». Ce panneau mobile était utilisé par le PFR afin de présenter aux élèves les ateliers de la matinée. Y figuraient ainsi le contenu de chaque atelier (par exemple "apprendre à reproduire des rythmes de perles ") et le lieu où allait se dérouler cet atelier (par exemple "à l'espace découverte du monde »). Afin de permettre aux élèves de verbaliser ces éléments sous la forme d'une phrase complète du type "À l'espace découverte du monde, nous allons apprendre à reproduire des rythmes de perles ", l'enseignante utilisait des référents sur le panneau (photo du lieu, support pédagogique pour les élèves). Néanmoins ce panneau ne montrait pas l'adulte sous la responsabilité de qui allait se dérouler l'atelier (l'enseignante ou l'Agent Territorial Spécialisé des Écoles 
Maternelles (ATSEM)). Le développement professionnel de l'activité dialogique du PFR porte précisément sur cet élément. C'est ce que montre l'extrait suivant (Extrait 4).

\section{Extrait 4 Contrôle-Accompagnement du second usage PFR - PFÉ}

\section{Notes d'observation du chercheur sur la pratique du PFÉ à partir de l'enregistrement vidéo}

Épic interroge les élèves sur un atelier de découpage. Il leur demande «Et pour l'atelier découpage, vous serez tout seul, avec maîtresse ou avec tatie Jojo ${ }^{2}$ ?"

\section{Extrait de l'échange}

PFR (53'3") : mais quels éléments ils ont pour savoir ?

PFÉ : de ? Non, répète ta question. Je n'ai pas suivi.

PFR : «à cet atelier vous serez avec... tout seul..."

PFÉ : là, je fais appel à leur mémoire. Je n'avais pas de photo, même pour l'atelier... tu vois ? Ils s'étaient déplacés, c'est qui, c'était Élise je crois. Pour aller montrer le coin, parce que je n’avais pas le coin en photo. PFR : c'est vrai que ce qui serait intéressant... je crois que je te l'ai suggéré l'autre fois... ce serait que sur ce panneau il y ait également la photo... comme ça, on pourrait verbaliser une phrase plus complexe «à l'espace langage... nous allons apprendre à... avec tatie Jojo ». Trop bien ! [...]

PFÉ : donc on mettrait la photo du coin, enfin de l'aménagement...

PFR : on aurait, le lieu, l'activité, et la personne avec qui ils vont travailler.

PFÉ : et la personne. Ce n’est pas une mauvaise idée.

Lors de cet échange, le PFR a évoqué le fait que la demande formulée par PFÉ aux élèves ne fournissait pas à ces derniers tous les éléments pour pouvoir répondre. Elle a proposé au PFÉ une nouvelle règle d'utilisation du panneau des ateliers permettant d'enrichir le langage des élèves. Cette formulation peut être synthétisée comme suit : [" (mettre) sur ce panneau (...) également la photo (de) la personne avec qui ils vont travailler» obtient pour résultats attendus "(de pouvoir) verbaliser une phrase plus complexe (...)»]. Outre le fait de trouver une solution au problème rencontré par le PFÉ, cet échange a conduit le PFR à réélaborer sa propre pratique de classe. C'est ce que révèle l'EAC du PFR sur cet extrait du contrôle-accompagnement du second usage (Extrait 5).

\section{Extrait 5 PFR - EAC - Contrôle-Accompagnement du second usage}

Chercheur (2h5'37') : que se passe-t-il ?

PFR : et ben, je cogite pour ma pratique aussi ! Parce que là, en fait, je parle d'un problème, entre guillemets, que je perçois. Elle (le PFÉ) leur demande (aux élèves) quelque chose, mais ils n’ont pas les éléments pour y répondre. En tout cas, là, moi en tant qu'observatrice, je me dis sur quoi ils vont s'appuyer pour y répondre? Elle argumente et puis là, dans ma tête je me dis, mais ouais, c'est vrai que ça pourrait être intéressant que dans le langage, que dans nos exigences, qu'elles soient d'un degré supplémentaire où on puisse non seulement dire " ce que l'on va apprendre ", " où ", mais aussi " avec qui ». Voilà, c'est plus par rapport au langage de l'élève. Donc en fait, de ce problème-là, ça m’ouvre des possibles quoi.

Cet extrait documente la réélaboration par le PFR du problème rencontré par le PFÉ et témoigne d'une mise en chantier conséquente de sa propre pratique, ("je me dis attends on va voir... de ce problème-là, je me dis tiens, peut-être que je pourrais..."), autour (a) de l'évolution de l'utilisation du panneau des apprentissages et (b) de l'élévation du degré d'exigence relativement au langage des élèves. Le développement de l'activité professionnelle de PFR peut être formulé au travers de la règle ["je cogite pour ma pratique" vaut pour "dans ma tête je me dis, mais ouais » ce qui obtient pour résultat "ça pourrait être intéressant que dans le langage, que dans nos exigences, quelles soient d'un degré supplémentaire où on puisse non seulement dire "ce que l'on va apprendre", "où", mais aussi "avec qui" "]. L'intérêt de cet extrait est de montrer que la pratique du PFÉ n'a pas constitué une simple exécution conforme aux règles enseignées, mais a été considérée par le PFR comme une variante féconde de la sienne. Ce résultat corrobore les conclusions de Yvon et Clot (2003), relatives au développement de l'activité

2 Tatie Jojo est l'une des deux ATSEM de la classe du PFÉ. Elle est chargée d'assister l'enseignante pour l'accueil et l'hygiène des jeunes élèves. Elle apporte également sa collaboration pour la réalisation d'ateliers avec des petits groupes d'élèves après avoir reçu les objectifs de la part de l'enseignante. 
professionnelle d'enseignant.e.s de philosophie échangeant à propos de leurs pratiques de présentation du travail à des élèves du second degré. Un développement du pouvoir d'agir a été constaté par les auteurs au cours et à l'issue d'une controverse stylistique entre ces professeurs. Le dispositif présenté ici rend compte précisément (a) d'un développement de l'action située du PFÉ, et non seulement d'un développement dialogique de son pouvoir d'agir, soit du rayon de "l'action possible » et (b) d'un développement en retour du pouvoir d'agir du PFR, mais pas encore de son pouvoir d'action effectif. En effet, l'issue positive donnée à l'enseignement ostensif de pratiques nouvelles par le PFR a également engendré chez cette dernière une réélaboration et un développement de son expérience dont on constate le compte rendu dialogique lors de l'EAC. Ces résultats permettent d'illustrer une autre forme de développement professionnel en rapport avec les dialogues de formation entre PFR et PFÉ. Nous ne pouvons documenter ici l'existence d'un développement de la pratique spécifique de PFR à la suite du développement de sa signification des règles enseignées au PFÉ, mais il nous apparaît évident qu'une étude complémentaire pourrait porter sur cet « effet en retour » du travail de formation sur le travail d'enseignement, soit de l'activité dialogique de formation sur l'activité d'enseignement au quotidien du PFR. Il serait opportun d'interroger également le changement de statut introduit par le dispositif de recherche qui produit lors de l'EAC une inversion de rôles : le PFÉ se transforme ici en une ressource collective du PFR pour penser/signifier autrement son propre enseignement au sein d'un dialogue où le chercheur devient l'interlocuteur principal. C'est ce dialogue qui se développe et qui pourrait être pris comme le point de départ pour la conception d'un autre dispositif de formation de formateur·rice·s, cette fois-ci du PFR. Beaucoup plus complet et moins linéaire, ce dispositif permettrait de concevoir la relation d'enseignement ostensif des règles comme un processus collectif dialectique, où les ressources partagées par un PFR enseignant ses pratiques serait considéré comme le point d'origine d'un enseignement en retour anticipé et non seulement constaté a posteriori de façon accidentelle, à l'insu du PFÉ.

\section{Discussion et conclusion}

Au début des années 1990, le système éducatif français a connu une évolution quant à la perception du rôle et par voie de conséquence aux modalités de formation des enseignant.e.s (Altet, 2012). Ce mouvement de professionnalisation s'est accompagné d'un "virage réflexif " (Tardif et al., 2012) faisant en même temps de l'enseignant-professionnel (Perrenoud, 1993) un "praticien réflexif " (Schön, 1994) susceptible d'apprendre seul par l'engagement d'une réflexion en cours et/ou sur sa pratique d'enseignement. Les résultats de la présente étude ainsi que ceux de travaux scientifiques récents invitent à émettre des réserves quant à la pertinence d'inscrire encore aujourd'hui le développement professionnel des enseignant.e.s au sein du seul paradigme du praticien réflexif. Pour accroitre l'efficacité de la formation continue, il semble pertinent de discuter ce paradigme dominant, notamment sur l'une de ses dimensions : celle de l'apprentissage du métier par la controverse stylistique au regard d'un problème professionnel.

La conception institutionnelle française du développement professionnel des enseignant.e.s suppose que l'apprentissage du métier se poursuit, notamment, par l'accumulation d'expériences construites au gré des circonstances professionnelles. Ce postulat renvoie au modèle constructiviste existentiel schönien (Charlier, 2012) selon lequel l'efficacité des conduites de l'enseignant·e serait due à des routines ainsi qu'à sa capacité à réfléchir dans l'action. Selon Schön (1994), ces routines seraient activées automatiquement en fonction d'éléments spécifiques de l'environnement dans lequel se déroule l'action. Confronté.e à un problème ou à une difficulté, l'enseignant.e serait amené.e à " apprendre " par une réflexion dans l'action lors de laquelle il ou elle expérimenterait de nouvelles conduites adaptées qui, à leur tour, concourraient à alimenter son référentiel de savoirs professionnels. Si la résolution individuelle de problèmes professionnels peut effectivement être source d'apprentissage pour les enseignant.e.s notamment pour faire réussir leurs élèves (Butler et Schnellert, 2012), il est intéressant de souligner que le premier résultat de la présente étude tend à montrer que, face à un problème professionnel, l'enseignant.e peut également être démuni.e et ne pas être en mesure de mobiliser les ressources individuelles nécessaires pour surmonter ce problème. En effet, la marionnette Épic était devenue dans la pratique antérieure du PFÉ un objet de distraction faisant obstacle à la concentration des élèves. Ce n'est que (a) par l'enseignement ostensif de la règle relative à l'utilisation d'une voix spécifique pour la marionnette ainsi que (b) par le suivi correct de cette règle dans le cours même du travail, que le PFÉ a réussi à surmonter le problème de la marionnette-peluche et à constater des résultats probants pour ses élèves. Ce résultat permet d'indiquer que tout apprentissage informel ne garantit pas une amélioration de l'efficacité de l'enseignement et peut même être contre-productif. Les travaux de recherche actuels portant sur les difficultés dans le travail 
enseignant (Murillo et Nunez-Moscoco, 2019) discutent également le rôle positif et constructif des difficultés professionnelles, qui engendrent parfois davantage de désillusions que de tâtonnements féconds. En ce sens, la " difficulté professionnelle ordinaire ", définie comme "l'incident ", "l'obstacle " ou encore " le problème " dans l'exercice du métier (Nunez-Moscoco et Murillo, 2017), serait porteuse de doutes cruciaux à propos des conditions du travail "bien fait " (Hélou et Lantheaume, 2008). Le travail empêché ou contrarié qui en résulte représenterait alors un risque pour la santé (Clot, 2008) des enseignant.e.s. Les mauvais résultats des élèves sont souvent perçus comme un échec professionnel et minent l'assurance du travail bien fait (Lantheaume, 2007).

Dans le prolongement des idées développées en amont, soulignons que notre étude corrobore le point de vue de Nunez-Moscoco et Murillo (2017) en proposant des solutions ancrées dans la formation continue des enseignant.e.s mobilisant notamment une collaboration effective au sein même de l'établissement scolaire (Hélou et Lantheaume, 2008). À ce titre, elle s'associe à d'autres travaux mettant en avant l'intérêt de la construction de territoires dits " apprenants " au sein des établissements scolaires et/ou de réseaux établis entre eux. Institutionnellement mis en avant sans être véritablement définis (Villani et Torossian, 2018), ces territoires peuvent être considérés comme apprenants lorsqu'ils recouvrent un espace source et ressource d'une communauté d'apprentissage professionnel (Leclerc, 2012). Autrement dit, ils sont apprenants, si et seulement si, ils offrent la possibilité aux membres de la communauté d'user du potentiel formateur de leur espace de travail en offrant les conditions d'un recouvrement plus ou moins exhaustif entre l'exercice du travail et la formation à celui-ci (Baeten et Simons, 2014). Bien évidement ce recouvrement, souvent seulement limité aux dimensions spatiales et temporelles, nécessite des conditions singulières de collaboration au sein même de la communauté professionnelle considérée, par exemple en termes de co-enseignement et/ou co-analyse entre pair.e.s (Huang et Shimizu, 2016 ; Soslau et al., 2019), mais aussi entre les membres de cette communauté et des partenaires extérieurs à celle-ci comme par exemple des formateur·rice-s et/ou chercheur·se·s (Ria et Moussay, 2014). À dimension technologique, certains travaux tentent, pour ainsi dire, d'aller un peu plus loin en essayant de mettre en exercice l'ensemble de ces conditions. C'est le cas, par exemple, des " Projets d'initiative locale adossée à la recherche " (PILAR) (Canet et Chaliès, 2020) ou encore des " espaces de travail collectif " (Moussay et Aknouche, 2020) au sein desquels la communauté professionnelle élargie aux formateur·rice.s et chercheur.se.s travaille à articuler au sein d'un même dispositif des temps " d'enseignement pour la formation " et des temps de "formation à l'enseignement".

Cette étude permet enfin de discuter les recherches menées au sein du même paradigme sur la formation des enseignant·e.s novices (Chaliès et Bertone, 2017). En effet, alors que dans les études menées en formation initiale le principe de l'alternance apparaît comme peu fécond (on y constate par exemple des avancées réflexives et dialogiques substantielles des enseignant.e.s novices en formation de terrain et une stagnation de leurs pratiques situées en classe (Chaliès et al., 2010)), la présente étude documente un développement conjoint de l'activité dialogique, de l'analyse réflexive à propos de l'action et des pratiques situées des enseignant.e.s participant. Ce développement accrédite l'hypothèse d'une fécondité des dispositifs de formation fondés sur le dialogue et la réflexivité entre pair·e.s expert·e.s, qui se révèlent pourtant peu efficaces dans le cas d'interactions dissymétriques entre tuteur-trice-s et novices en formation initiale. Bien que des initiatives, telles que le " peer-teaching ", le "peer-coaching", ou le " peer-mentoring ", soient prises pour favoriser la multiplicité des interactions d'entraide entre pair.e.s débutant.e.s dans le cadre de la formation initiale (pour une revue : Chaliès et al., 2009), notre étude permet à son échelle d'investiguer la spécificité de deux " grandes " modalités d'application du principe de l'alternance en formation d'adultes, fondées sur des interactions le plus fréquemment dissymétriques (en formation initiale) versus symétriques (en formation continue entre pair-e.s). Les résultats de la présente recherche permettent de supposer l'hypothèse qu'en formation continue entre pair.e.s, la négociation de l'objet de formation à un niveau local au sein de l'établissement scolaire est un élément déterminant pour le succès du dispositif de développement professionnel. L'enjeu d'approfondissement de ce champ de recherche est à la fois épistémique et technologique. Comme souligné en amont, il trouve un écho actuel au sein des " territoires apprenants " (Delavet et Olivier, 2014), qui font aujourd'hui l'objet d'une attention tant institutionnelle que scientifique.

Pour conclure, il apparaît qu'en prenant en compte les pratiques ordinaires des enseignant.e.s comme substrats d'une démarche de recherche transformative et en s'inscrivant au sein de l'établissement scolaire, le dispositif support à notre étude a pu pallier certains écueils d'une formation continue régulièrement déconnectée de la pratique effective des enseignant·ess, tout en plaçant le collectif au cœur du développement professionnel. Ces caractéristiques technologiques concourent ainsi favorablement au développement de la réflexivité de l'enseignant.e face aux problèmes professionnels. Plus en avant, c'est ici le rôle de la réalisation effective, aménagée ou pas, du travail qui est ici mis en exergue. Notre étude, bien qu'exploratoire, invite en

2021 RSSE 42 (3), DOI $10.24452 / \mathrm{sjer.43.1.12}$ 
effet à considérer autrement les temps de réalisation du travail pour faire en sorte qu'ils soient davantage intégrés au dispositif de formation et étayent, en pleine continuité avec les temps de formation, le flux expérientiel des formé.e.s.

\section{Bibliographie}

Altet, M. (2012). Les compétences de l'enseignant-professionnel : entre savoirs, schèmes d'action et adaptation, le savoir analyser. Dans L. Paquay, M. Altet, E. Charlier et P. Perrenoud (dir.), Former des enseignants professionnels (p. 43-57). Éditions De Boeck Supérieur.

Baeten, M., et Simons, M. (2014). Student teachers' team teaching : Models, effects and conditions for implementation. Teaching and Teacher Education, 41, 92-110.

Bell, J., et Dale, M. (1999). Informal learning in the workplace. Department for Education and Employment Research Report, 134.

Bertone, S. (2016). La force des règles dans l'apprentissage du métier enseignant. Éditions universitaires européennes.

Bertone, S., et Chaliès, S. (2015). Construire un programme de recherche technologique sur la formation des enseignants : choix épistémologiques et théoriques. Activités, 12(2), 53-72. http://www.activites.org/v12n2/V12n2.pdf

Borko, H., Koellner, K., Jacobs, J., et Seago, N. (2011). Using video representations of teaching in practice-based professional development programs. ZDM Mathematics Education, 43(1), 175-187.

Bouysse, V. (2018). Enseigner en maternelle, expérience particulière pour le professeur des écoles ou métier spécifique ? Administration et éducation, 158(2), 77-82.

Butler, D. L., et Schnellert, L. (2012). Collaborative inquiry in teacher professional development. Teaching and Teacher Education, $28(8), 1206-1220$.

Canet, F., et Chaliès, S. (2020). L’accompagnement du mouvement professionnel : le cas des PILAR. Diversité, 197, 152-156.

Chaliès, S., et Bertone, S. (dir.). (2008). L'enseignement. Éditions Revue EPetS.

Chaliès, S., et Bertone, S. (2017). Cultural anthropology and teacher education. Dans M. Peters et J. Stickney (dir.), A Companion to Wittgenstein on Education: Pedagogical Investigations (p. 659-673). Springer.

Chaliès, S., Bruno, F., Méard, J., et Bertone, S. (2010). Training preservice teachers rapidly: the need to articulate the training given by university supervisors and cooperating teachers. Teaching and Teacher Education, 26, 764-774.

Chaliès, S., Cartaud, S., Escalié, G., et Durand, M. (2009). Note de synthèse - D’une utilité discutée à une utilité éprouvée du tutorat en formation initiale des enseignants : la nécessité d'une formation des tuteurs. Recherche et Formation, 61, 85-129.

Charlier, E. (2012). Former des enseignants-professionnels pour une formation continuée articulée à la pratique. Dans L. Paquay, M. Altet, E. Charlier et P. Perrenoud (dir.), Former des enseignants professionnels (p. 43-57). Éditions De Boeck Supérieur.

Clot, Y. (2008). Travail et pouvoir d'agir. PUF.

Consuegra, E., Engels, N., et Struyven, K. (2014). Beginning teachers' experience of the workplace learning environment in alternative teacher certification programs: A mixed methods approach. Teaching and Teacher Education, 42, 79-88.

Delavet, T., et Olivier M.-F. (2014). La culture au cour des apprentissages. Un nouveau projet pour l'école : stratégie culturelle et territoire apprenant. ESF.

Ermeling, B. A., et Yarbo, J. (2016). Expanding instructional horizons: A case study of teacher team-outside expert partnership. Teachers Colleges Record, 118(2), 1-48.

Félix, C. (2014). Du travail des " collectifs » à de nouvelles modalités de formation professionnelle : l'histoire du GAP. Questions Vives, 21. https ://questionsvives.revues.org/1510.

Fraser, C. A. (2010). Continuing professional development and learning in primary science classrooms. Teacher Development: An international Journal of Teachers' Professional Development, 14, 85-106.

González, G., Deal, J. T., et Skultety, L. (2016). Facilitating teacher learning when using different representations of practice. Journal of Teacher Education, 67(5), 447-466.

Grau, V., Calcagni, E., Preiss, D. D., et Ortiz, D. (2017). Teachers' professional development through university-school partnerships: Theoretical standpoints and evidence from two pilot studies in Chile. Cambridge Journal of Education, 47(1), $19-36$.

Hélou, C, et Lantheaume, F. (2008) Les difficultés au travail des enseignants. Exception ou part constitutive du métier ? Recherche et Formation, 57, 65-78.

Huang, R., et Shimizu, Y. (2016). Improving teaching, developing teachers and teacher educators and linking theory and practice through lesson study in mathematics: an international perspective. ZDM, 48(4), 393-409.

IGEN. (2017). Rapport $n^{\circ}$ 2017-032. La scolarisation en petite section de maternelle. Repéré à https://www.education.gouv.fr/ la-scolarisation-en-petite-section-de-maternelle-6500

Lakatos, I. (1994). Histoire et méthodologie des sciences. PUF.

Lantheaume, F. (2007). L'activité enseignante entre prescription et réel : ruses, petits bonheurs, souffrance. Éducation et sociétés, 19(1), 67-81.

Lave, J., et Wenger, E. (1991). Situated learning: Legitimate peripheral participation. Cambridge University Press.

Leclerc, M. (2012). Communauté d'apprentissage professionnelle : Guide à l'intention des leaders scolaires. Presses de l'Université du Québec.

Little, J. W. (1993). Teachers' professional development in a climate of education reform. Educational Evaluation and Policy Analysis, 15, 129-151.

Little, J. W. (2007). Teachers' accounts of classroom experience as a resource for professional learning and instructional decision making. Dans P. A. Moss (dir.), Evidence and decision making. 2007 NSSE Yearbook, 106(1), 217-240. Blackwell Synergy.

Lomos, C., Hofman, R. H., et Bosker, R. J. (2011). Professional community and student achievement - a meta-analysis. School Effectiveness and School Improvement, 22(2), 121-148. 
Moussay, S., et Aknouche, S. (2020). Une autre façon de penser l'accompagnement en recherche. Diversité, 197, 90-94.

Moussay, S., et Blanjoie, V. (2016). Intervenir pour aider les enseignants dans la prise en charge des élèves décrocheurs. Questions vives, 25 . https://journals.openedition.org/questionsvives/1887

Murillo, A., et Nunez-Moscoso, J. (2019). Les recherches sur la difficulté dans le travail enseignant : approches et résultats. Carrefours de l'éducation, 47, 225-255.

Nelson, K. (2008). Wittgenstein and contemporary theories of word Learning. New Ideas in Psychology, 4, 1-13.

Nunez-Moscoso, J., et Murillo, A. (2017). La difficulté dans le travail enseignant : un thème de recherche aux objets pluriels. Penser l'éducation, 40, 59-86.

OCDE. (2019). Résultats de TALIS 2018 (Volume I) : Des enseignants et chefs d'établissement en formation à vie. OCDE.

Ogien, A. (2007). Les formes sociales de la pensée. La sociologie après Wittgenstein. Armand Colin.

Perrenoud, P. (1993). Formation initiale des maitres et professionnalisation du métier. Sciences de l'Éducation, XIX(1), 59-76.

Postholm, M. B. (2018). Teachers' professional development in school: A review study. Cogent education, 5(1), 1-22.

Poulson, L., et Avramidis, E. (2003). Pathways and possibilities in professional development: case studies of effective teachers of literacy. British Educational Research Journal, 29, 543-560.

Ria, L., et Lussi Borer, V. (2015). Laboratoire d'analyse vidéo de l'activité enseignante au sein des établissements scolaires : enjeux, méthodes et effets sur la formation des enseignants. Dans L. Ria (dir.), Former les enseignants au $21^{e}$ siècle. Volume 1: Établissement formateur et vidéoformation (p. 101-117). Éditions De Boeck.

Ria, L., et Moussay, S. (2014). "Change laboratories" within secondary schools: towards accompaniment and support of teachers in the appropriation of the new reforms in priority education. Dans F. Nyhamn et T. N. Hopfendeck (dir.), From Political Decisions to Change in the Classroom (p. 78-95). CIDREE Yearbook.

Schön, D. A. 1994. Le praticien réflexif. A la recherche du savoir caché dans l'agir professionnel. Éditions Logiques.

Soslau, E., Gallo-Fox, J., et Scantlebury, K. (2019). The promises and realities of implementing a coteaching model of student teaching. Journal of Teacher Education, 70(3), 265-279.

Stein, M. K., Smith, M. S., et Silver, E. A. (1999). The development of professional developers: Learning to assist teachers in new settings in new ways. Harvard Educational Review, 69(3), 237-269.

Stoll, L., Bolam, R., McMahon, A., Wallace, M., et Thomas, S. (2006). Professional learning communities: A review of the literature. Journal of Educational Change, 7(4), 221-258.

Suchaut, B. (2008). Le rôle de l'école maternelle dans les apprentissages et la scolarité des élèves. Conférence pour l'A.G.E.M. Bourges, France. https://halshs.archives-ouvertes.fr/halshs-00240399

Talérien, S., et Bertone, S. (2018). La transmission explicite des connaissances ordinaires des enseignants expérimentés comme ressource de la formation continue. SHS Web Conf., 52. https ://doi.org/10.1051/shsconf/20185201004

Tardif, M., Borgès, C., et Malo, A. (dir.). (2012). Le virage réflexif en éducation. Éditions De Boeck Supérieur.

Theureau, J. (2006). Le cours d'action. Méthode développée. Octarès.

Vescio, V., Ross, D., et Adams, A. (2008). A review of research on the impact of professional learning communities on teaching practice and student learning. Teaching and Teacher Education, 24(1), 80-91.

Villani, C., et Torossian, C. (2018). Rapport. 21 mesures pour l'enseignement des mathématiques. Repéré à https://www.education. gouv.fr/21-mesures-pour-l-enseignement-des-mathematiques-3242

Wittgenstein, L. (2004). Recherches philosophiques. Gallimard.

Wood, K., Jaidin, H., Jawawi, R., Perera, J. S. H. Q., Salleh, S., Shahrill, M., et Sithamparam, S. (2017). How and what teachers learn from collaborative professional development. International Journal of Lesson and Learning Studies, 6(2), 151-168.

Yvon, F., et Clot, Y. (2003). Apprentissage et développement dans l'analyse du travail enseignant. Pratiques psychologiques, 1, $19-35$.

Mots-clés : Développement professionnel ; enseignant expérimenté ; formation continue ; règles de métier ; pratique ordinaire 


\section{Förderung der beruflichen Entwicklung durch Unterstützung der Reflexivität in der Weiterbildung: eine Fallstudie im Kindergarten}

\section{Zusammenfassung}

Diese Fallstudie basiert auf einem kulturanthropologischen Forschungsprogramm, und schlägt ein forschungsbasiertes Ausbildungsprogramm vor, das formale Voraussetzungen für die effektive Vermittlung von Regeln im Rahmen der alltäglichen Unterrichtspraxis zwischen zwei erfahrenen Lehrern festlegt, die im selben Kindergarten arbeiten. Um sich beruflich weiterzuentwickeln, durchliefen die beiden Lehrer (der eine in der Rolle des «Peer-Trainers» und der andere in der Rolle des "geschulten Peers») drei Trainingselemente: (i) Unterstützung des Peer-Trainers/der Peer-Trainerin bei der Vermittlung einer klareren Vorstellung von den Regeln, die für seine/ihre Praxis gelten und die zu vermitteln sind, (ii) direkte Vermittlung dieser Regeln durch den PeerTrainer/die Peer-Trainerin an den/die geschulte(n) Peer und (iii) zwei praktische Umsetzungen durch den/die geschulte(n) Peer, jeweils gefolgt von einer begleitenden Aktivität in Form einer Supervision durch den/die Peer-Trainer/in. Die erzielten Ergebnisse zeigen (a) das Erlernen einer neuen Regel durch den geschulten Peer, die es ihm ermöglicht, eine berufliche Schwierigkeit zu lösen, und (b) die berufliche Entwicklung des PeerTrainers durch die Begleitung des geschulten Peers.

Schlagworte: Berufliche Entwicklung; erfahrene Lehrer/innen; Weiterbildung; Regeln; gewohnte Praxis

\section{Stimolare lo sviluppo professionale facilitando la riflessività nella formazione continua: uno studio di caso alla scuola dell'infanzia}

Riassunto

Questo studio di caso propone, a partire da un programma di ricerca in antropologia culturale, un dispositivo di formazione che cerca di creare le condizioni formali per un'efficace trasmissione delle pratiche didattiche ordinarie tra due insegnanti esperti che lavorano nella stessa scuola dell'infanzia. Per produrre il loro sviluppo professionale, i due insegnanti (uno nel ruolo di "peer trainer" e l'altro nel ruolo di "peer learner") sono stati sottoposti a tre momenti di formazione : (i) l'accesso preventivo da parte del peer trainer ad una visione più chiara delle regole che reggono la sua pratica da trasmetetre (ii) l'insegnamento "ostensivo" di queste regole da parte del peer trainer al peer learner e (iii) due implementazioni da parte del peer learner seguite da un accompagnamento sotto forma di supervisione da parte del peer trainer. I risultati ottenuti mostrano (a) l'apprendimento di una nuova regola da parte del peer learner che gli permette di risolvere una difficoltà professionale e (b) lo sviluppo professionale del peer trainer che realizza l'insegnamento del peer learner.

Parole chiave: Sviluppo professionale; insegnante esperto; formazione continua; regole, pratica ordinaria

\section{Generating professional development by supporting reflexivity in continuing education: a case study in kindergarten}

\section{Abstract}

This case study proposes, based on an anthropocultural research program, a research-based training scheme that creates the formal conditions for the effective transmission of the rules of ordinary pedagogical practice between two experienced teachers working in the same nursery school. In order to produce their professional development, the two teachers (one in the role of "peer trainer" and the other in the role of "trained peer") underwent three training episodes : (i) prior access by the peer trainer to a clearer vision of the rules governing his practice to be transmitted (ii) ostensive teaching of these rules by the peer trainer to the trained peer and (iii) two implementations by the trained peer followed each time by an accompanying activity in the form of supervision of the peer trainer. The results obtained show (a) the learning of a new rule by the trained peer enabling him/her to solve a professional difficulty as well as (b) the professional development of the peer trainer following his/her accompaniment of the practice of the trained peer.

Key words: Professional development; experienced teacher; continuing education; professional rules; ordinary practice 
Stéphane Talérien. Il est Docteur en Sciences de l'éducation et de la formation. Chercheur associé au laboratoire Ingénierie, Sport, Santé et Environnement (IRISSE EA 4075) à l'Université de La Réunion (France), son objet d'étude traite de la formation professionnelle continue des enseignant.e.s. Ses travaux s'inscrivent au sein du paradigme anthropoculturel posant une conception "horizontale " et "locale " de la formation comme partage et transmission entre pairs de pratiques circonstanciées jugées pertinentes par les enseignant·e.s eux-mêmes. Le développement professionnel des enseignant.e.s y est étudié par la focale de l'analyse de leur activité professionnelle.

Université de La Réunion, 117 avenue du Général Ailleret, 97430 Le Tampon, La Réunion.

E-Mail : jean-stephane.talerien@univ-reunion.fr

Sébastien Chaliès. Il est Professeur des Universités à l'Institut National Supérieur du Professorat et de l'Éducation de Toulouse Occitane Pyrénées (Université de Toulouse - France). L'ensemble de son travail scientifique a pour finalité la construction et le développement d'un "programme de recherche » singulier mené en sciences de la formation d'adultes. Mené en anthropologie culturaliste, ce programme porte sur la construction du sujet professionnel en formation. D’un point de vue épistémologique, ce programme emprunte à la philosophie analytique, à la sociologie de la connaissance et à l'anthropologie culturelle. Les principales problématiques travaillées au cœur de ce programme sont relatives au principe de l'alternance entre travail et formation au travail, à la place de l'ostension/prescription et de la réflexivité en formation, à la construction de la santé au et par le travail ou encore à la créativité professionnelle.

INSPE de Toulouse Occitanie Pyrénées - Site de Rangueil, 118 Route de Narbonne, F-31078 Toulouse.

E-Mail : sebastien.chalies@univ-tlse2.fr

Stefano Bertone. Il est Professeur des Universités à l'Institut National Supérieur du Professorat et de l'Éducation de La Réunion (Université de La Réunion - France). Les recherches menées par Stefano Bertone, portent sur l'évaluation et la conception des dispositifs de formation d'adultes dans les milieux de l'enseignement et de l'entrainement sportif. Elles se déploient au sein d'un " programme de recherche technologique " ancré sur le cadre conceptuel de l'anthropologie culturaliste. Elles s'inspirent de la philosophie analytique de Wittgenstein et des théories de l'action collective. Les objets d'étude portent sur le principe de l'alternance entre travail/entrainement et formation au travail. Elles interrogent la place de la réflexivité dans le développement de l'activité professionnelle des enseignant·e.s et des entraineur.euse.s sportifs et visent des applications en termes d'ingénierie de contexte, répondant à des commandes institutionnelles.

INSPE de La Réunion - Site du Tampon, 117 Avenue du Général Ailleret, 97430 Le Tampon, La Réunion.

E-Mail : stefano.bertone@univ-reunion.fr 